\title{
Failures of Retrieval and Comparison Constrain Change Detection in Natural Scenes
}

\author{
Andrew Hollingworth \\ Yale University
}

\begin{abstract}
In a change detection paradigm, a target object in a natural scene either rotated in depth, was replaced by another object token, or remained the same. Change detection performance was reliably higher when a target postcue allowed participants to restrict retrieval and comparison processes to the target object (Experiment 1). Change detection performance remained excellent when the target object was not attended at change (Experiment 2) and when a concurrent verbal working memory load minimized the possibility of verbal encoding (Experiment 3). Together, these data demonstrate that visual representations accumulate in memory from attended objects as the eyes and attention are oriented within a scene and that change blindness derives, at least in part, from retrieval and comparison failure.
\end{abstract}

People spend most of their waking lives within environments that typically contain a great deal of visual detail and many constituent objects. The visual complexity of natural environments necessitates selective visual processing of local scene regions by movements of the eyes and attention (see Henderson \& Hollingworth, 1998, 1999a). ${ }^{1}$ Figure 1 shows a typical eye movement scan pattern on a scene during $20 \mathrm{~s}$ of viewing. Note that the eyes sequentially visit many local objects and that few fixations are directed to empty or noninformative regions, such as the walls and floor. Similar eye movement characteristics are found when participants perform everyday tasks, such as preparing food (Hayhoe, 2000; Land, Mennie, \& Rusted, 1999). Given the local and serial nature of scene perception, if the visual system is to construct a global representation of a scene, information from previously fixated and attended objects must be retained in memory and integrated with information from subsequently selected objects. Understanding scene perception therefore depends on understanding the characteristics of visual memory.

The role of visual memory in scene representation has proven a controversial issue. On the one hand, a prominent class of theory holds that visual memory is transient, with little retention and accumulation of visual information from previously selected objects (Ballard, Hayhoe, \& Pelz, 1995; Irwin, 1992a, 1992b; Irwin \& Andrews, 1996; Irwin \& Zelinsky, 2002; O'Regan, 1992; O'Regan \& Nöe, in press; O'Regan, Rensink, \& Clark, 1999; Rensink, 2000; Rensink, O’Regan, \& Clark, 1997; Simons, 1996; Simons \& Levin, 1997; Wolfe, 1999). On the other hand, Hollingworth and Henderson (2002; see also Henderson \& Hollingworth,

Andrew Hollingworth, Department of Psychology, Yale University.

This research was supported by National Institute of Mental Health Grant R03 MH65456. Aspects of this study were reported at the 42nd Annual Meeting of the Psychonomic Society, Orlando, Florida, November 2001. I thank John Henderson and Dan Simons for helpful comments on an earlier version of the manuscript.

Correspondence concerning this article should be addressed to Andrew Hollingworth, who is now at the Department of Psychology, University of Iowa, 11 Seashore Hall E, Iowa City, Iowa 52242-1407. E-mail: andrew-hollingworth@uiowa.edu in press-b) have proposed that visual information from attended objects is retained robustly after the withdrawal of attention and accumulates in memory during scene viewing, based on evidence of accurate visual memory for previously attended objects (Hollingworth \& Henderson, 2002; Hollingworth, Williams, \& Henderson, 2001). A parallel debate can be found in the literature on visual search, focusing on the question of whether participants have memory for previously examined items (e.g., Horowitz \& Wolfe, 1998; Shore \& Klein, 2000). Because it concerns the basic issue of how humans represent the visual environment around them, the debate over visual memory has wide implications that extend throughout cognitive science.

The claim that visual memory is transient has been driven largely by the phenomenon of change blindness. Participants have demonstrated a surprising degree of difficulty in detecting changes to scenes when detection depends on visual memory, such as when a change is introduced during a saccade (e.g., Grimes, 1996; Henderson \& Hollingworth, 1999b) or across a brief interstimulus interval (ISI; e.g., Rensink et al., 1997). According to visual transience theories, change blindness occurs because visual scene representations are impoverished. Scene representations are impoverished because the visual representation of an attended object

\footnotetext{
${ }^{1}$ During free viewing, the spatial orientation of the eyes and visual attention are functionally coupled. Prior to a saccadic eye movement, visual attention is shifted from the currently fixated object to the saccade target (Henderson, Pollatsek, \& Rayner, 1989; Rayner, McConkie, \& Ehrlich, 1978). The shift of attention to the saccade target appears to be mandatory and exclusive, as participants cannot attend to one object while preparing a saccade to a different object (Deubel \& Schneider, 1996; Hoffman \& Subramaniam, 1995; Kowler, Anderson, Dosher, \& Blaser, 1995; Shepherd, Findlay, \& Hockey, 1986). In addition, events that capture visual attention, such as an abrupt visual onset, also capture the eyes (Theeuwes, Kramer, Hahn, \& Irwin, 1998). Thus, when discussing perceptual selection in real-world scenes, I make the assumption that the eyes go where attention goes. Of course, attention can be oriented covertly (without a subsequent eye movement to the attended object) when saccades are strategically inhibited (e.g., Posner, Snyder, \& Davidson, 1980), but such laboratory conditions are uncharacteristic of typical scene perception, in which participants rarely have any cause to inhibit eye movements.
} 


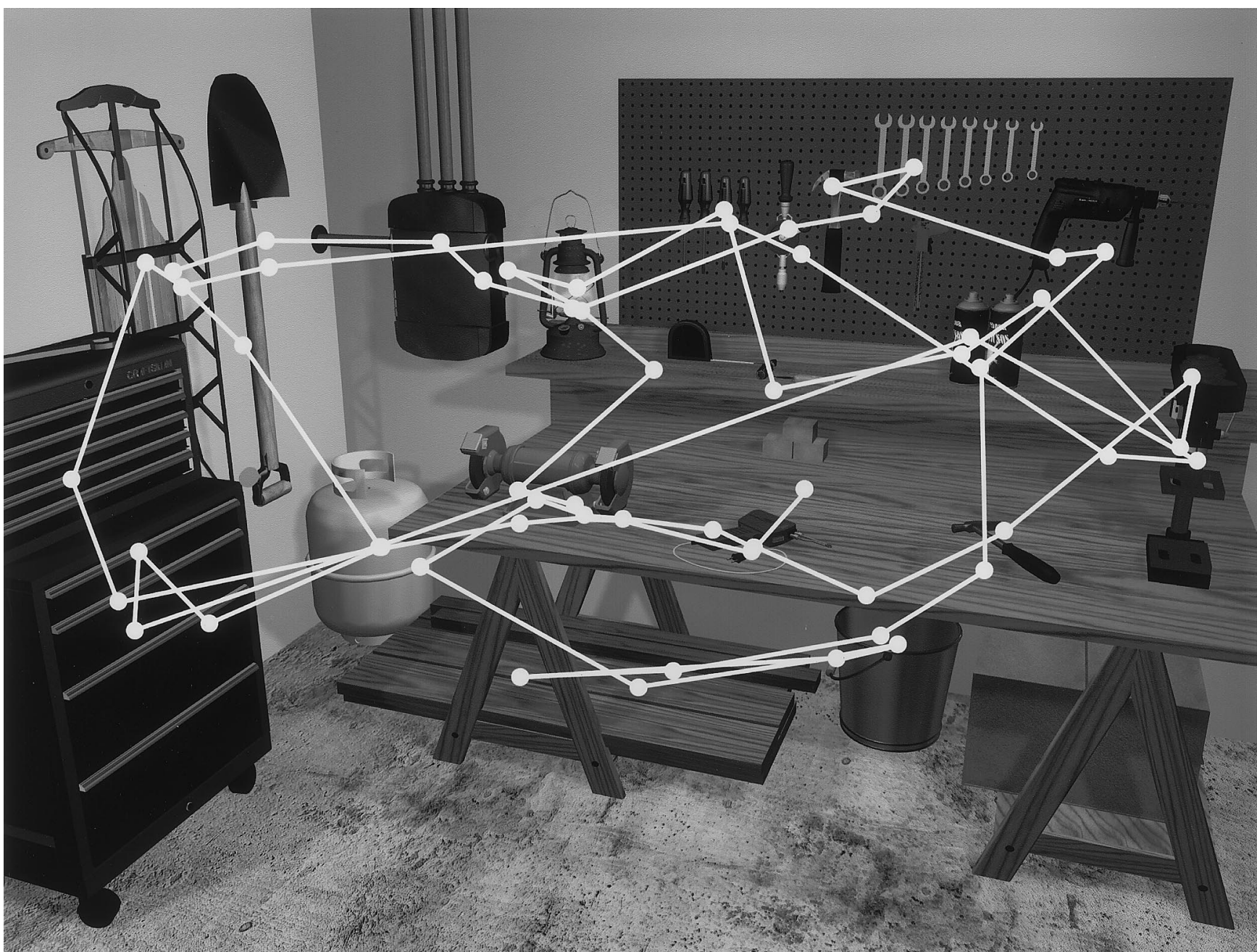

Figure 1. Eye movement scan path (from a different study) of a single participant on a scene. The scene was visible for $20 \mathrm{~s}$ and was viewed in preparation for a memory test. Lines represent saccades; dots represent fixations. (A color version of this figure is available in the online version of this article, which is part of the PsycARTICLES database.)

decays very quickly after the withdrawal of attention from that object. In particular, visual memory for local objects is proposed to be either nonexistent (O'Regan, 1992; O'Regan \& Nöe, in press), limited to the currently attended object (Rensink, 2000; Rensink et al., 1997; Wolfe, 1999), or limited to the currently attended object plus the two or three most recently attended objects (Irwin, 1992a; Irwin \& Andrews, 1996; Irwin \& Zelinsky, 2002). In addition to such transient forms of visual memory, the scene representation may be composed of other, more stable information, such as the semantic gist of the scene (e.g., bedroom), the spatial layout of visual objects, or the identities of recognized objects. Instead of relying on memory for the visual characteristics of a scene, the visual system exploits the stability of the visual world, which can be sampled and resampled as ongoing tasks demand. The world itself serves as an "outside memory" (O'Regan, 1992). The stronger versions of the visual transience hypothesis produce the empirical claim that attention must be oriented to an object when it changes to detect the change (Rensink, 2000; Rensink et al., 1997).
In contrast to these proposals, Hollingworth and Henderson (2002) and Hollingworth, Williams, and Henderson (2001) have provided evidence that representations from previously attended objects can be retained robustly in visual memory. In these experiments, eye movements were monitored while participants viewed real-world scenes, and visual changes were introduced on the basis of eye position. The computer waited until the participant had fixated a target object in the scene (to ensure it had been attended prior to the change), and the target object was then changed during a subsequent saccade to a different (nontarget) object in the scene. Because visual attention is automatically and exclusively allocated to the goal of a saccade prior to the initiation of that eye movement (e.g., Hoffman \& Subramaniam, 1995), the target object was not attended when the change occurred: Attention had shifted to the nontarget object that was the goal of the eye movement. Thus, the paradigm tested memory for previously attended objects.

The target object was changed either by rotating it $90^{\circ}$ in depth or by replacing it with another object from the same basic-level 
category (a token change). Neither change altered the gist of the scene, the spatial layout of the scene, or the basic-level identity of the target object. Yet, participants were able to detect these changes on a significant proportion of trials. In addition, participants were able to make accurate two-alternative, forced-choice orientation and token decisions for previously attended objects (greater than $80 \%$ correct). Accurate discrimination performance was observed even when many fixations on other objects intervened between target fixation and test. Finally, accurate discrimination performance was observed on a long-term memory test conducted 5-30 min after initial viewing. Thus, these experiments suggest that visual memory is far from transient; visual representations sufficient to make subtle judgments can be retained robustly across multiple shifts of the eyes and attention within a scene, and even across different intervening scenes.

To account for these results, Hollingworth and Henderson (2002) proposed that visual representations are preserved after the withdrawal of attention and are accumulated to form a relatively detailed scene representation. It is clear that visual representations stored over the course of seconds are not sensory (or iconic) in nature (Averbach \& Coriell, 1961; Sperling, 1960; see Irwin, $1992 b$, for a review), but abstracted (or higher level) visual representations, such as those supporting object recognition, can be of sufficient specificity to code individual exemplars and object orientation (Tarr \& Bülthoff, 1998). In this visual memory theory of scene representation (Hollingworth \& Henderson, 2002), as the eyes and attention are oriented within a scene, higher level visual representations are formed for attended objects. The visual representation of an object is then indexed to a position within a spatial representation of the scene and is transferred from visual shortterm memory (VSTM) to long-term memory (LTM), where it is retained and accumulates with visual representations from other previously attended objects. During online viewing, visual object representations are reactivated when attention is allocated back to the object's position, influencing ongoing perceptual processing of the scene and supporting accurate memory performance, such as change detection.

But there remains a puzzle. If visual scene memory can be both accurate and robust, why would change blindness ever be observed? That is, even with evidence of robust visual memory, it is important to understand the origin of the change blindness phenomenon that led researchers to argue for visual transience in the first place. Hollingworth and Henderson (2002) proposed two possibilities for how change blindness may arise despite the capability to retain and accumulate visual representations in real-world scenes. First, changes may be missed because the relevant object was not focally attended before the change was introduced. The transfer of visual information into memory is strongly dependent on the allocation of visual attention and of the eyes (Averbach \& Coriell, 1961; Currie, McConkie, Carlson-Radvansky, \& Irwin, 2000; Henderson \& Hollingworth, 1999b; Hollingworth \& Henderson, 2002; Hollingworth, Schrock, \& Henderson, 2001; Irwin, 1992a; Irwin \& Gordon, 1998; McConkie \& Currie, 1996; Nelson \& Loftus, 1980; Schmidt, Vogel, Woodman, \& Luck, 2002; Sperling, 1960). Thus, in change detection paradigms, if an object has not yet been attended and fixated when it is changed, participants should very rarely detect that change, despite the capability to encode and retain visual information from that object and despite accurate and robust memory for other objects that have been attended.

The second main possibility, investigated in the present study, is that even if visual information sufficient to detect a change has been encoded, that information may not be retrieved and compared with current perceptual information from the changed image (Hollingworth \& Henderson, 2002; Simons, 2000; Simons, Chabris, Schnur, \& Levin, 2002). ${ }^{2}$ The traditional assumption in the change detection literature has been that explicit change detection performance is a direct reflection of visual memory capacity: If a change is detected and reported, information relevant to detecting the change had been stored in visual memory; if not, then the relevant information had not been stored (e.g., Rensink et al., 1997; see Pashler, 1988, for a formalization of this assumption; see Simons, 2000, for a discussion of alternative models). This assumption has received support from VSTM studies using arrays of relatively simple stimuli (Becker, Pashler, \& Anstis, 2000; Vogel, Woodman, \& Luck, 2001). In these studies, the target object was either specified in the test image (i.e., postcued) or not specified. If retrieval and comparison limitations constrain change detection performance, then participants should have benefited from the ability to restrict retrieval and comparison to the target object. Yet, neither study found a change detection advantage for the postcue condition compared with the no-postcue condition, suggesting that memory capacity was the primary source of limitations on change detection.

In contrast to abstract arrays of simple stimuli, however, change detection in real-world scenes may benefit from postcuing, because real-world scenes contain far more visual information relevant to the change detection decision. In particular, the set of potentially changing objects is always specified in studies using abstract arrays; the test array can be analyzed exhaustively by comparing each item with memory for the initial array. In realworld scenes, the set of potentially changing objects is far less clear (see Figure 1), and the changed target may not be compared with memory on some proportion of trials.

Three strands of data provide initial evidence that change blindness may derive, to some extent, from retrieval and comparison failure and thus that change detection in natural scenes would benefit from target postcuing in the test image. The first is evidence of preserved visual memory in the absence of explicit report of change (Fernandez-Duque \& Thornton, 2000; Hayhoe, Bensinger, \& Ballard, 1998; Henderson \& Hollingworth, in press-a; Hollingworth \& Henderson, 2002; Hollingworth, Williams, \& Henderson, 2001; Williams \& Simons, 2000). ${ }^{3}$ For example, Hollingworth, Williams, and Henderson (2001) found that when participants failed to report a change in a real-world scene, fixation durations on changed objects were significantly elevated compared

\footnotetext{
${ }^{2}$ Although retrieval failure and comparison failure could be separate causes of change blindness, current evidence, including the present study, does not allow them to be analyzed separately.

${ }^{3}$ Recent work has questioned whether effects of change on indirect measures, such as fixation duration or response latency, reflect a different form of representation than that supporting explicit change detection (Mitroff, Simons, \& Franconeri, 2002). However, the fact that effects of unreported changes appear on indirect measures, and thus that explicit report underestimates visual memory, is not disputed.
} 
with those in a no-change control condition. These results demonstrate that change detection failure can occur despite the retention of a visual representation from the changed target, and they therefore falsify the assumption that explicit change detection provides an exhaustive measure of visual memory. Such evidence raises the possibility that with an effective retrieval cue, participants could bring additional information to bear on the change detection decision, increasing detection performance.

Second, Hollingworth and Henderson (2002) and Henderson and Hollingworth (1999b) found that the detection of changes to previously attended objects was often delayed until the changed object happened to be refixated later in viewing. After the change, participants clearly had a memory representation sufficient to detect that change (because it was detected later), but this representation was not compared with current perceptual information for a significant amount of time (detection latency was approximately $5 \mathrm{~s}$ on average). A plausible interpretation of this effect is that a visual representation was encoded when the target was initially attended, remained in an inactive state as subsequent objects were inspected, and was retrieved when cued by refixation of the target, leading to change detection.

Finally, evidence of retrieval and comparison failure in change detection paradigms comes from a recent study by Simons et al. (2002). An experimenter, posing as an athlete and holding a red and white basketball, asked a passerby for directions to the gym. During the interaction, a crowd of collaborators intervened between the interlocutors, and the basketball was covertly removed. Simons et al. found that some participants who did not report noticing a change nevertheless could describe the absent basketball when asked specifically. Although this method cannot isolate visual memory, the fact that participants could sometimes report the visual characteristics of the removed basketball suggests that they may have retained a visual representation of the basketball that was retrieved only when the participant received an effective retrieval cue.

The present study had two goals. First, I sought to test whether change blindness in complex, natural scenes derives from limitations on retrieval and comparison processes by introducing a target postcue manipulation. Second, I sought to provide converging evidence that visual memory representations are retained robustly after the withdrawal of attention by using a new method to manipulate attention in scenes: abrupt visual onsets.

\section{Experiment 1}

A change detection paradigm was used in which an initial image of a 3-D scene was presented for $20 \mathrm{~s}$, followed by an abrupt object onset and offset within the scene, a pattern mask, and a test scene (see Figure 2). The initial viewing duration was chosen to be long enough to ensure that the target would be fixated and attended prior to the change on the vast majority of trials. (In Hollingworth \& Henderson, 2002, using a similar set of stimuli, the target object was fixated within $20 \mathrm{~s}$ of viewing on $98 \%$ of trials.) In the test scene, a single target object was either changed or remained the same. Two types of changes were possible: a rotation, in which the target was rotated $90^{\circ}$ in depth, and a token change, in which the target was replaced by another object from the same basic-level category. Figure 3 illustrates the change manipulations.
To examine whether change blindness can derive from retrieval and comparison failure, I introduced a postcue manipulation in the test scene. On half the trials, the target object was specified in the test scene by an arrow cue, in which case the task was to determine whether the specified object had changed. On the other half, the target object was not specified, in which case the task was to determine whether any object in the scene had changed. The latter method is characteristic of studies demonstrating change blindness. Yet, if change blindness is caused to some significant extent by retrieval and comparison failure, detection performance should be higher in the postcue condition, when participants can limit retrieval and comparison to the target object.

In previous studies (Hollingworth \& Henderson, 2002; Hollingworth, Williams, \& Henderson, 2001), object changes were completed during a saccade to a different object in the scene to ensure that attention was not allocated to the target object when it changed. The present study used a different method of controlling the allocation of attention. A neon green dot was flashed briefly in the scene prior to the appearance of the mask. In Experiment 1, this onset cue was noninformative, appearing in a region of the scene not occupied by any object that could plausibly be a target. The abrupt appearance of a new visual object should capture attention (Jonides, 1981; Yantis \& Jonides, 1984), and thus the target object should not be attended when the change occurs. In addition, abrupt visual onsets capture attention even when they are known to be invalid on all trials (Remington, Johnston, \& Yantis, 1992). And, although participants can resist attentional capture when they can direct attention to a different object immediately before an onset (e.g., Theeuwes, 1991; Yantis \& Jonides, 1990), such strategic preparation could not be easily accomplished in the present paradigm, because participants were actively examining the scene when the onset occurred and did not know where the onset would occur.

If visual representations are reliably retained from previously attended objects, as held by visual memory theory, and if limitations on retrieval and comparison are alleviated by means of the postcue, then change detection performance should be very accurate in the postcue condition. In contrast, visual transience theories claiming that visual memory is limited to the currently attended object (e.g., Rensink, 2000) predict chance performance in all conditions, because the target would not be attended when the change occurred. And, of course, this view predicts no effect of postcue, because it holds that there is no visual memory whose retrieval would be influenced. The present paradigm does not directly test Irwin and colleagues' (Irwin, 1992a; Irwin \& Andrews, 1996; Irwin \& Zelinsky, 2002) claim that visual memory can retain two or three previously attended objects, as the onset cue manipulation can ensure only that the target is not focally attended when the change occurs. Evidence directly relevant to this theory is reviewed in the General Discussion.

\section{Method}

Participants. A group of 12 participants from the Yale University community completed the experiment. They either received course credit in introductory psychology or were paid. All participants had normal or corrected-to-normal vision.

Stimuli. Forty-two initial scene images were computer rendered from 3-D wire-frame models. Each model depicted a typical, human-scaled environment (e.g., kitchen or playground). A target object was chosen 

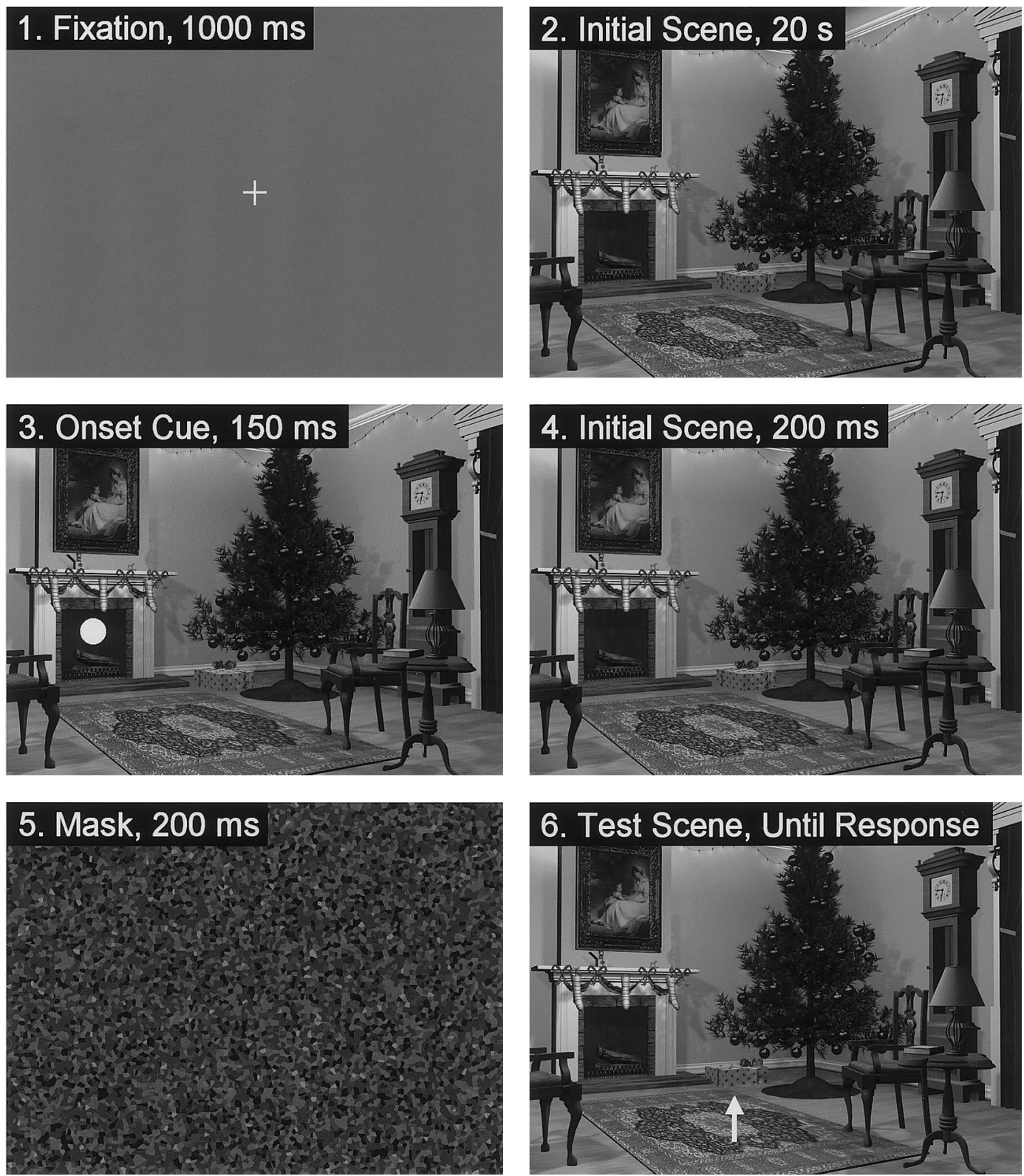

Figure 2. Sequence of events in a trial. In this example, the gift is the target object, the onset cue is invalid, and the target is rotated $90^{\circ}$ in depth and is postcued in the test scene. (Scene stimuli in this study were presented in color. A color version of this figure is available in the online version of this article, which is part of the PsycARTICLES database.)

within each model. The rotation and token-change images were created by rerendering the scene after the target object had been either rotated $90^{\circ}$ in depth or replaced by another object from the same basic-level category. The objects for token changes were chosen to be approximately the same size as the initial target object. In the changed scenes, the 3-D graphics software automatically corrected for changes in occlusion, lighting, and so on. At a viewing distance of $80 \mathrm{~cm}$, scene images subtended $16.9^{\circ} \times 22.8^{\circ}$ of visual angle. Target objects subtended $3.33^{\circ}$ on average along the longest dimension in the picture plane. The onset cue was a neon green disk with a diameter of $1.15^{\circ}$. The postcue was a neon green arrow subtending $2.22^{\circ}$ in length, and it pointed unambiguously to the target on all trials in the postcue condition. The mask was made up of a patchwork of small colored shapes and was the same size as the scene stimuli.
Apparatus. The stimuli were displayed at a resolution of $800 \times 600$ pixels, in 24-bit color, on a 17-in. $(43.18 \mathrm{~cm})$ Sony Trinitron monitor with a refresh rate of $85 \mathrm{~Hz}$. The initiation of image presentation was synchronized to the monitor's vertical refresh. Responses were collected by the computer keyboard. The presentation of stimuli and collection of responses was controlled by an $850-\mathrm{MHz}$ Pentium III computer. The room was dimly illuminated by a low-intensity light source.

Procedure. Participants were tested individually. Each participant was given a written description of the experiment along with a set of instructions. Participants were informed that they would view a series of scene images and would have to determine whether a change had been introduced on each trial. The nature of the two possible changes was described. After review of the instructions, participants were seated in front of the computer 


\section{Same}

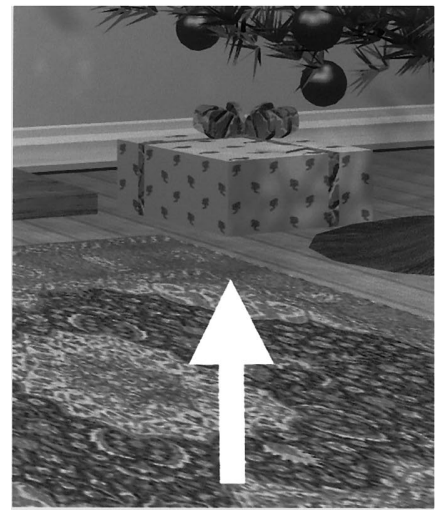

Rotation

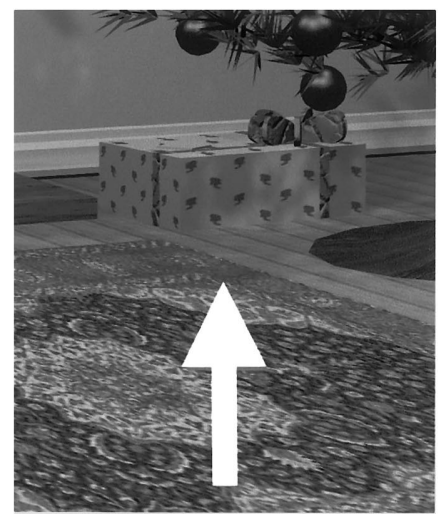

Token change

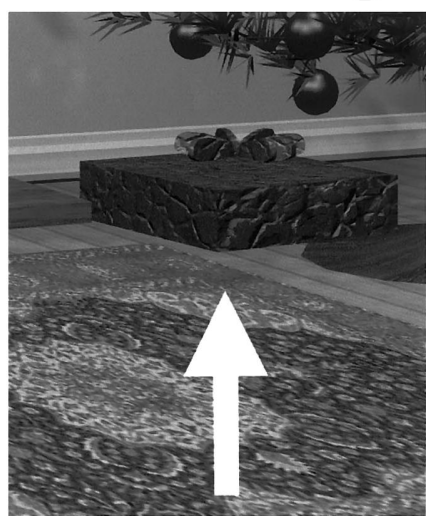

Figure 3. Detail of one scene stimulus illustrating the change manipulations (with the arrow postcue). (Scene stimuli in this study were presented in color. A color version of this figure is available in the online version of this article, which is part of the PsycARTICLES database.)

display at a viewing distance of $\sim 80 \mathrm{~cm}$. No head restraint was used, but participants were asked to remain in the same position throughout the experiment.

Participants pressed a pacing button to initiate each trial. Then, a white fixation cross on a gray field was displayed for $1,000 \mathrm{~ms}$. This was followed by the initial scene presentation for $20 \mathrm{~s}$, the onset cue within the scene for $153 \mathrm{~ms}$, the initial scene again for $200 \mathrm{~ms}$, the pattern mask for $200 \mathrm{~ms}$, and finally the test scene, which remained visible until response. Participants pressed either the 1 key to indicate a "same" response or the 0 key to indicate a "changed" response.

Participants first completed a practice session of six trials, one in each of the conditions created by the 2 (postcue or no postcue) $\times 3$ (change type: same, rotation, or token change) factorial design. The scenes used for the practice trials were not used in the experimental session. In the experimental session, participants viewed all 42 scenes, seven in each of the six conditions. Across participants, each scene item appeared in each condition an equal number of times. The postcue factor was blocked, and block order was manipulated between participant groups. Because block order did not produce a reliable main effect on detection performance, nor did it interact with other factors, all analyses are collapsed across block order. Within each block, trial order was randomly determined for each participant. The entire session lasted approximately $45 \mathrm{~min}$.

\section{Results}

In this and in subsequent experiments, three measures of detection performance are reported: $A^{\prime}$, percentages correct, and reaction times (RTs) for all responses. $A^{\prime}$ is a nonparametric signal detection measure with a functional range of .5 (chance) to 1.0 (perfect sensitivity; Grier, 1971). For each participant in each change condition, $A^{\prime}$ was calculated using the mean hit rate when the target changed and the mean false alarm rate when it did not. For example, $A^{\prime}$ for postcued rotations was calculated using the participant's hit rate in the postcue-rotation condition and the false alarm rate in the postcue-same condition. Because $A^{\prime}$ corrects for potential differences in response bias in the percentage correct data, it forms the primary data for interpreting these experiments. Reported analyses treated participant as a random effect. ${ }^{4}$

$A^{\prime}$ analysis. Mean $A^{\prime}$ in each of the change conditions is displayed in Figure 4 . The postcue factor produced a reliable main effect, $F(1,11)=11.78, p<.01$. Sensitivity to change was higher in the postcue condition (.89) than in the no-postcue condition (.75). The main effect of change type was not reliable $(F<1)$, nor was the interaction between postcue and change type, $F(2,22)=$ 2.70, $p=.13$.

Percentage correct analysis. Percentage correct data are presented in Table 1. The change conditions (rotation and token change) and same condition were analyzed separately. In the change conditions, there was a reliable main effect of postcuing, $F(1,11)=10.04, p<.01$. Participants responded correctly $77.4 \%$ of the time in the postcue condition and $61.3 \%$ of the time in the no-postcue condition. The effect of change type was not reliable $(F<1)$, nor was the interaction between postcue and change type, $F(1,11)=2.24, p=.16$. In the same condition, the postcuing effect was not significant, $F(1,11)=2.31, p=.15$, although the numerical trend was in the same direction as in the change conditions.

$R T$ analysis. Mean RTs for all responses are presented in Table 1 . In the change conditions, there was a reliable main effect of postcuing, $F(1,11)=26.51, p<.001$. Consistent with the $A^{\prime}$ and percentage correct data, participants responded more quickly in the postcue condition (3,002 $\mathrm{ms})$ than in the no-postcue condition $(5,171 \mathrm{~ms})$. The effect of change type was not reliable, $F(1$, 11) $=1.56, p=.24$, nor was the interaction between postcue and change type $(F<1)$. Similarly, in the same condition, mean RT was reliably shorter in the postcue condition $(3,615 \mathrm{~ms})$ than in the no-postcue condition $(6,462 \mathrm{~ms}), F(1,11)=45.94, p<.001$. The $\mathrm{RT}$ data for correct responses produced the same pattern of results.

\footnotetext{
${ }^{4}$ For the percentage correct and RT data, analyses treating scene item as a random effect were also conducted but are not reported. In each of the three experiments, all effects that were statistically reliable by subjects were also reliable by items. Item analyses cannot be conducted over $A^{\prime}$ data, because sensitivity must be calculated independently for each participant.
} 


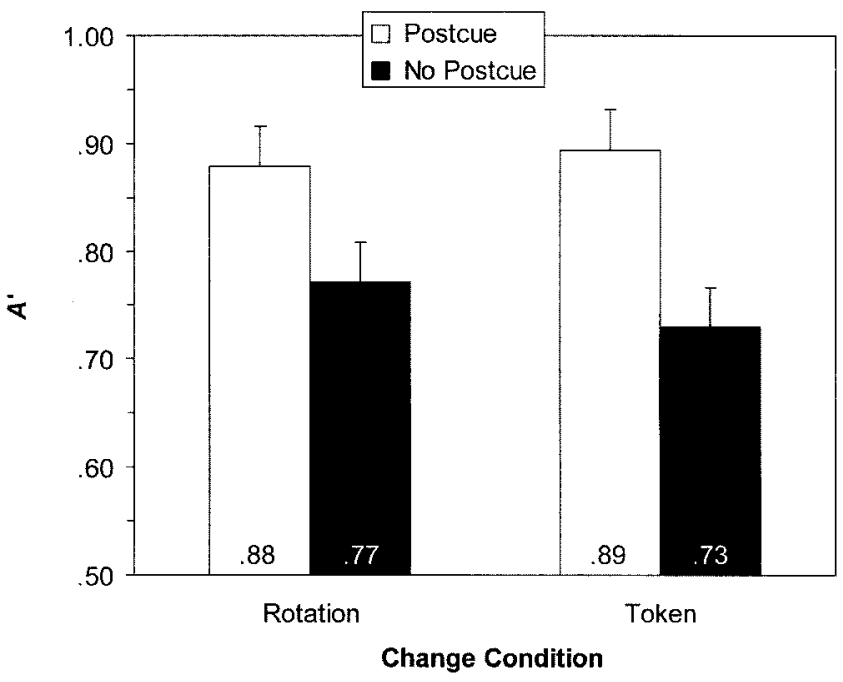

Figure 4. Experiment 1: Mean $A^{\prime}$ as a function of postcue and change type. Error bars represent $95 \%$ confidence intervals based on the interaction error term.

\section{Discussion}

The data from Experiment 1 demonstrate that change blindness derives, at least in part, from limitations on retrieval and comparison processes. First, change detection performance was well above chance in both the postcue and no-postcue conditions, demonstrating that visual representations of objects can be retained robustly after the withdrawal of attention. Second, change detection performance was reliably higher when a postcue allowed participants to limit retrieval and comparison processes to the target. Thus, retrieval and comparison failure is likely to have played a significant role in participants' dramatic inability to detect changes to real-world scenes in previous studies (e.g., Grimes, 1996; Rensink et al., 1997) and, to some extent, in the no-postcue condition of this study. This conclusion is strengthened by the RT data in Experiment 1. Participants spent more than $2 \mathrm{~s}$ longer on average to respond in the no-postcue condition than in the postcue condition, presumably because they had to assess multiple objects in the former condition but only one in the latter. In summary, when limitations on retrieval and comparison were lifted, change detection performance for visual changes was very accurate indeed, consistent with the prediction of visual memory theory.

\section{Experiment 2}

In Experiment 1, onset cues were noninformative, appearing on a region of the scene that could not plausibly be a target object. Thus, Experiment 1 depended on the assumption that attention was captured involuntarily by the onset cue. Although it is unlikely that participants could have avoided attention capture in Experiment 1, it is also the case that involuntary attention capture has been demonstrated in paradigms significantly different from the present study. Therefore, Experiment 2 used a converging method to control attentional allocation that did not depend on the assumption of involuntary capture. Trials were divided evenly between valid onset cues (i.e., the green disk appeared on the target object) and invalid onset cues (i.e., the green disk appeared on a nontarget object in the scene). Given that there are many potentially changing objects in each scene (see the scene stimuli in Figures 1 and 2, which are of representative complexity), a cue that is valid $50 \%$ of the time should provide strong incentive to direct attention to the cued object. Invalid trials then provide evidence regarding change detection performance for objects that were not currently attended when the change occurred. As in Experiment 1, three change conditions were used: same, rotation, and token change. In addition, all trials contained a postcue arrow specifying the target object.

The valid onset cue should allow participants to encode additional target information immediately before the change. Thus, detection performance in the valid onset condition is likely to be higher than that in the invalid onset condition (Scholl, 2000). However, if visual representations are reliably retained after the withdrawal of attention, performance in the invalid condition should remain accurate.

\section{Method}

Participants. A group of 12 new participants from the Yale University community completed the experiment for pay. All participants had normal or corrected-to-normal vision.

Stimuli and apparatus. The stimuli were same as in Experiment 1. The apparatus was the same as in Experiment 1, with the following exceptions: Responses were collected using a serial button box, and viewing distance was fixed at $80 \mathrm{~cm}$ by means of a forehead rest.

Procedure. The procedure was similar to that in Experiment 1. Participants first completed a practice session of six trials, one in each of the conditions created by the 2 (valid onset cue or invalid onset cue) $\times 3$ (change type: same, rotation, or token change) factorial design. In the experimental session, participants viewed all 42 scenes, seven in each of the six conditions. Trial order was randomly determined for each participant, with valid and invalid onset trials randomly intermixed. The entire session lasted approximately $45 \mathrm{~min}$.

\section{Results}

$A^{\prime}$ analysis. Mean $A^{\prime}$ in each of the change conditions is displayed in Figure 5. The onset cue validity factor produced a reliable main effect, $F(1,11)=10.81, p<.01$. Sensitivity to change was higher in the valid onset condition (.94) than in the invalid onset condition (.87). The main effect of change type was

Table 1

Experiment 1: Mean Percentages Correct and Mean Reaction Times for All Responses as a Function of Postcue and Change Type

\begin{tabular}{lccc}
\hline & \multicolumn{3}{c}{ Change condition } \\
\cline { 2 - 4 } Condition and measure & Same & Rotation & Token change \\
\hline $\begin{array}{l}\text { Postcue } \\
\text { \% correct }\end{array}$ & 83.3 & 75.0 & 79.8 \\
$\quad \begin{array}{l}\text { Reaction time (ms) } \\
\text { No postcue }\end{array}$ & 3,615 & 2,825 & 3,180 \\
$\quad \%$ correct & 74.9 & 65.5 & 57.2 \\
$\quad$ Reaction time (ms) & 6,462 & 4,949 & 5,393 \\
\hline
\end{tabular}




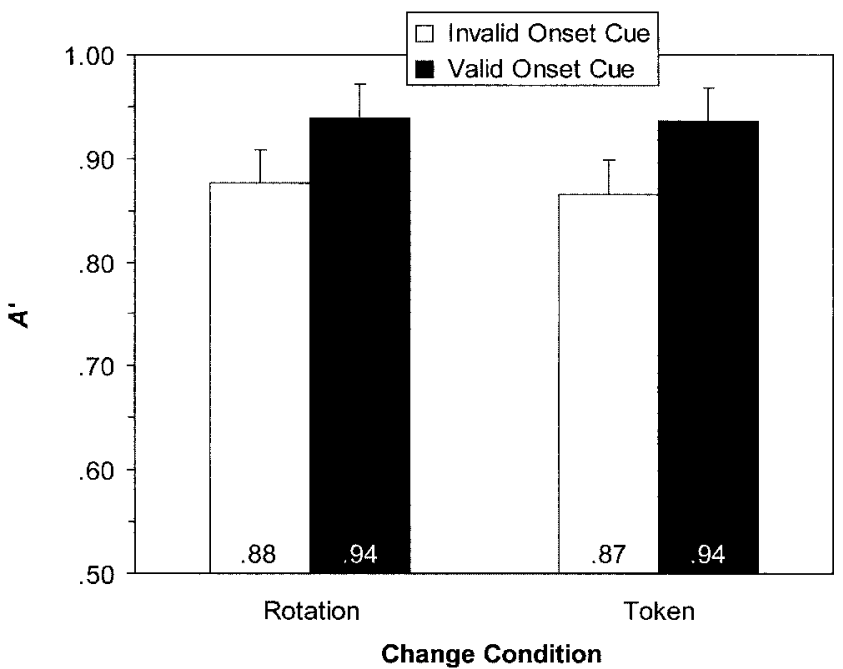

Figure 5. Experiment 2: Mean $A^{\prime}$ as a function of onset validity and change type. Error bars represent $95 \%$ confidence intervals based on the interaction error term.

not reliable $(F<1)$, nor was the interaction between onset validity and change type $(F<1)$.

Percentage correct analysis. Percentage correct data are presented in Table 2. In the change conditions, there was a reliable main effect of onset validity, $F(1,11)=19.08, p<.005$. Participants responded correctly $92.3 \%$ of the time in the valid condition and $68.4 \%$ of the time in the invalid condition. The effect of change type was not reliable $(F<1)$, nor was the interaction between onset validity and change type $(F<1)$. In the same condition, the onset validity effect was not reliable $(F<1)$.

$R T$ analysis. Mean RTs for all responses are presented in Table 2. In the change conditions, there was a reliable main effect of onset validity, $F(1,11)=57.72, p<.001$. Consistent with the $A^{\prime}$ and percentage correct data, participants responded more quickly in the valid condition $(2,445 \mathrm{~ms})$ than in the invalid condition $(3,972 \mathrm{~ms})$. The effect of change type was not reliable $(F<1)$, nor was the interaction between onset validity and change type $(F<1)$. In the same condition, there was a nonreliable trend toward shorter RTs with valid cuing, $F(1,11)=2.55, p=.14$. The $\mathrm{RT}$ data for correct responses produced the same pattern of results.

\section{Discussion}

In Experiment 2, the validity of the onset cue was manipulated (50\% valid and 50\% invalid) to ensure that participants had incentive to orient attention to the cued object. In addition, all target objects were postcued in the test scene. Detection sensitivity was reliably higher in the valid onset condition than in the invalid onset condition, presumably because additional target information was encoded immediately before the change in the valid condition. The onset dot was presented for $153 \mathrm{~ms}$, followed by $200 \mathrm{~ms}$ of additional viewing of the initial scene. Thus, there was ample time (353 ms) for participants to shift attention to the peripheral onset cue and to encode additional information from the cued object before the change. The onset cue validity effect demonstrates that participants did indeed orient attention to the cued object; there- fore, one can be confident that in the invalid trials, participants were not attending the target object when the change occurred. Yet, detection sensitivity in the invalid condition remained accurate and at levels similar to those found in Experiment 1. This result provides strong evidence that changes to previously attended objects can be detected accurately, consistent with visual memory theory.

\section{Experiment 3}

The goal of this study was to specifically investigate visual memory during scene viewing; therefore, it is important to ensure that verbal encoding cannot account for successful change detection in Experiments 1 and 2. Evidence of a role for verbal encoding in visual change detection paradigms is mixed. Vogel et al. (2001) found little effect of a concurrent verbal working memory load on visual change detection performance. However, Simons (1996) found reduced ability to detect identity changes to picture arrays with verbal shadowing than without. The token changes in the present study do not alter the basic-level identity of the target, and rotations do not alter the identity of the target at all. So it is not entirely clear how to map the Simons result onto the present paradigm. However, it is at least possible that verbal encoding contributed to the successful change detection performance in Experiments 1 and 2.

In Experiment 3, on half the trials a concurrent verbal working memory and articulatory suppression task was added to the change detection paradigm. In this verbal load condition, four random digits were presented at the beginning of each trial, and participants were required to repeat the digits aloud throughout the trial Onset cues in Experiment 3 were noninformative (as in Experiment 1), allowing all trials to contribute to the analysis of verbal load. All target objects were postcued in the test scene. If accurate change detection performance derives from verbal encoding, performance should be significantly worse when such encoding is impeded by the maintenance of a verbal working memory load.

\section{Method}

Participants. A group of 12 new participants from the Yale University community completed the experiment for pay. All had normal or correctedto-normal vision.

Stimuli and apparatus. The stimuli were same as in Experiments 1 and 2. The apparatus was the same as in Experiment 2.

Table 2

Experiment 2: Mean Percentages Correct and Mean Reaction Times for All Responses as a Function of Onset Validity and Change Type

\begin{tabular}{lccc}
\hline & \multicolumn{3}{c}{ Change condition } \\
\cline { 2 - 4 } Condition and measure & Same & Rotation & Token change \\
\hline $\begin{array}{l}\text { Invalid onset } \\
\quad \% \text { correct }\end{array}$ & 89.3 & 70.5 & 66.3 \\
$\quad \begin{array}{l}\text { Reaction time (ms) } \\
\text { Valid onset }\end{array}$ & 3,544 & 4,099 & 3,846 \\
$\quad \%$ correct & 85.8 & 92.9 & 91.8 \\
$\quad$ Reaction time (ms) & 3,181 & 2,419 & 2,471 \\
\hline
\end{tabular}


Procedure. The procedure was the same as in Experiments 1 and 2, except for the addition of a verbal working memory load on half the trials. In this experiment, the initial screen instructing participants to press a button to start the next trial also contained memory-load instructions. Either the word none was displayed for the no-load condition, or four randomly chosen digits were displayed for the memory-load condition. In the former condition, participants proceeded as in Experiments 1 and 2. In the latter condition, participants began repeating the four digits aloud and continued to repeat the digits throughout the trial. Participants were instructed to repeat the digits without interruption or pause. The experimenter monitored digit repetition to ensure that participants complied. After each trial in this condition, the participant wrote down the digits on a sheet of paper. Report accuracy was very high, with only one incorrectly reported digit across the entire experiment.

Participants first completed a practice session of six trials, one in each of the conditions created by the 2 (no load or memory load) $\times 3$ (change type: same, rotation, or token change) factorial design. In the experimental session, participants viewed all 42 scenes, seven in each of the six conditions. Trial order was determined randomly for each participant, with memory-load and no-load trials randomly intermixed. The entire session lasted approximately $45 \mathrm{~min}$.

\section{Results}

$A^{\prime}$ analysis. Mean $A^{\prime}$ in each of the change conditions is displayed in Figure 6. The main effect of memory load was not reliable, $F(1,11)=1.09, p=.32$, nor was the main effect of change type $(F<1)$. There was, however, a reliable interaction between these factors, $F(1,11)=5.82, p<.05$. Examining each of the contrasts, there was no effect of memory load for the rotation condition $(F<1)$, but performance was reliably higher with a memory load than without in the token-change condition, $F(1,11)=9.30, p<.05$.

Percentage correct analysis. Percentage correct data are presented in Table 3 . In the change conditions, there was neither a reliable main effect of memory load $(F<1)$, nor a reliable main effect of change type $(F<1)$. The interaction between these variables, however, approached reliability, $F(1,11)=4.73, p=$ .05. Examining each of the contrasts, there was no effect of

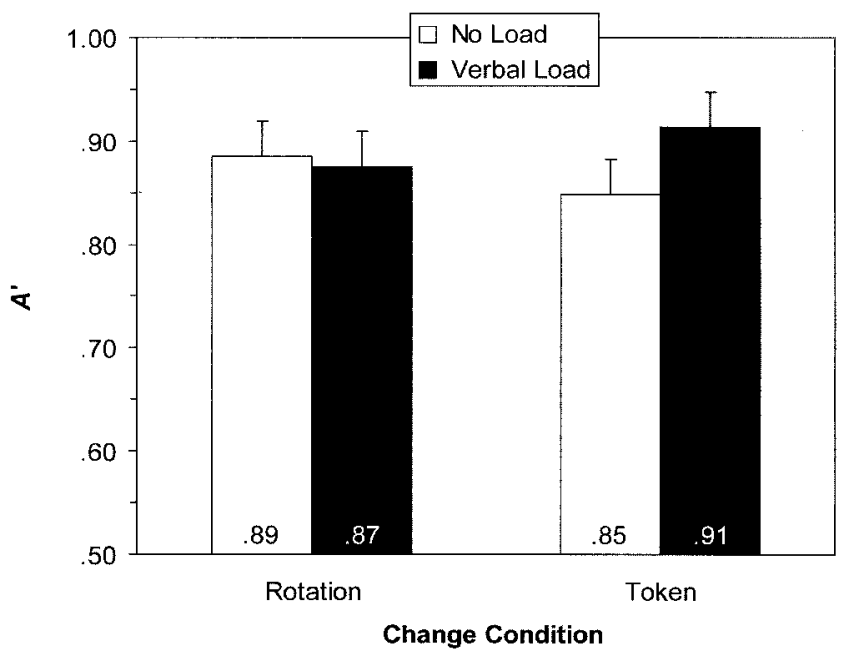

Figure 6. Experiment 3: Mean $A^{\prime}$ as a function of verbal memory load and change type. Error bars represent $95 \%$ confidence intervals based on the interaction error term.
Table 3

Experiment 3: Mean Percentages Correct and Mean Reaction Times for All Responses as a Function of Verbal Memory Load and Change Type

\begin{tabular}{lccc}
\hline & \multicolumn{3}{c}{ Change condition } \\
\cline { 2 - 4 } Condition and measure & Same & Rotation & Token change \\
\hline No load & & & \\
$\quad \%$ correct & 78.6 & 82.2 & 71.4 \\
$\quad \begin{array}{l}\text { Reaction time (ms) } \\
\text { Verbal memory load }\end{array}$ & 4,822 & 4,747 & 4,831 \\
$\quad \%$ correct & 84.7 & 78.6 & 83.4 \\
$\quad$ Reaction time (ms) & 5,048 & 4,549 & 4,742 \\
\hline
\end{tabular}

memory load for the rotation condition $(F<1)$, but performance was marginally higher with a memory load than without in the token-change condition $F(1,11)=2.32, p=.15$. In the same condition, there was a nonreliable trend in the direction of better performance in the memory-load condition, $F(1,11)=2.67, p=$ 13 .

$R T$ analysis. Mean RTs for all responses are presented in Table 3. In the change conditions, neither the memory load nor the change type factor produced a reliable effect $(F<1)$, and these factors did not interact $(F<1)$. In the same condition, the effect of memory load was not reliable $(F<1)$. The RT data for correct responses produced the same pattern of results.

\section{Discussion}

The addition of a verbal working memory load and articulatory suppression did not reduce change detection performance: Change detection was excellent in both the memory-load and no-load conditions. In fact, for token changes, $A^{\prime}$ performance was actually higher in the memory-load condition, although the source of this effect is not clear. Nevertheless, these results provide clear evidence that accurate change detection performance in the present study (and in previous studies: Hollingworth \& Henderson, 2002; Hollingworth, Williams, \& Henderson, 2001) derived from visual, not verbal, representation.

The results of this experiment are consistent with Vogel et al. (2001), who also failed to observe a decrement in change detection performance with the addition of a verbal memory load. In addition, the working memory load in this experiment was considerably more difficult than that used in Vogel et al. Why, then, did Simons (1996) find that verbal shadowing interfered with the detection of object replacement in arrays of pictures? One possibility is that Simons's change manipulation-substitution of one picture in an array with another from a different category-may have led participants to adopt a verbal strategy of encoding object identity. Such a strategy would not be useful for detecting the more subtle visual changes used in the present study. In addition, participants in the Simons study had to monitor for three different types of change on each trial (substitution, position switch, and configuration change). The addition of a difficult shadowing task to an already difficult change detection task may have left few resources available to monitor for object substitutions. 


\section{General Discussion}

In this study, I investigated the nature of visual memory for objects in natural scenes. First, I tested the possibility that change detection failure (or change blindness) may be caused by limitations on retrieval and comparison processes, consistent with evidence of robust visual memory and with visual memory theory (Hollingworth \& Henderson, 2002). Second, I sought converging evidence for the retention of visual object representations after the withdrawal of attention, using abrupt visual onsets to control attention. The paradigm displayed an initial scene for $20 \mathrm{~s}$, an abrupt object onset and offset within the scene (onset cue), a mask, and then a test scene that was either the same as the initial scene or the same except for a change to a single target object. Experiment 1 used a postcue manipulation to test whether limitations on retrieval and comparison constrain change detection performance. Change detection was more accurate when participants could limit retrieval and comparison to the target (postcue condition) than when they could not (no-postcue condition). In addition, with the benefit of a postcue, change detection performance was generally very high. In Experiment 2 the validity of the onset cue was manipulated to ensure that memory was tested for previously attended objects. The advantage for valid onset cues demonstrated that participants shifted attention to the cued object, but change detection for previously attended objects in the invalid condition remained high. In Experiment 3, change detection performance was not reduced by the introduction of a verbal working memory load and articulatory suppression, demonstrating that accurate change detection derives from visual, not verbal, memory.

There now exists substantial evidence from this and converging studies that visual memory for objects in scenes can be quite accurate. The present data replicate earlier demonstrations of preserved visual memory after the withdrawal of attention (Hollingworth \& Henderson, 2002; Hollingworth, Williams, \& Henderson, 2001) and extend that work by using abrupt visual onsets to control attention. There appears to be no way to reconcile these results with the proposal that a visual object representation is stable only when attention is directed to the represented object (Rensink, 2000; Rensink et al., 1997; Wolfe, 1999) or with the empirical claim that attention must be oriented to an object when it changes to detect the change (Rensink et al., 1997). Although the strong version of the visual transience hypothesis is not supported by this study, the present data do not necessarily rule out the possibility that visual memory is limited to the currently attended object plus the two or three most recently attended objects (Irwin \& Andrews, 1996; Irwin, 1992a). The onset cue ensured that participants were not attending the target when it changed, but it could not ensure that the target was not one of the two or three objects attended prior to the onset cue. However, Hollingworth and Henderson (2002) demonstrated that visual object representations can be retained over many intervening fixations on other objects and even across multiple scene stimuli. Neither of these results can be explained by a view in which visual memory is limited to three or four objects.

Thus, despite evidence of change blindness from other studies (e.g., Grimes, 1996; Rensink et al., 1997), visual scene representation is not impoverished, and visual object representations do not necessarily disintegrate at, or even soon after, the withdrawal of attention. Visual memory can be robust. This conclusion is sup- ported by converging evidence from other experimental paradigms investigating visual memory, including viewpoint effects in novel object recognition (e.g., Tarr, Bülthoff, Zabinski, \& Blanz, 1997; Tarr, Williams, Hayward, \& Gauthier, 1998), negative priming of nonsense shapes (DeSchepper \& Treisman, 1996), and most directly, highly accurate LTM for large numbers of pictures (Nickerson, 1965; Shepard, 1967; Standing, 1973; Standing, Conezio, \& Haber, 1970).

The visual memory debate has also arisen within the literature on visual search. This version of the debate began with evidence from Horowitz and Wolfe (1998) that search slopes were not increased when the positions of array items were periodically scrambled. From these and related data (Horowitz \& Wolfe, 2001; Wolfe, Klempen, \& Dahlen, 2000), they argued that visual search is amnesic for previously examined items: Visual object representations disintegrate as soon as attention is withdrawn (Wolfe, 1999). However, considerable evidence has been collected demonstrating memory for various forms of visual information during search: the spatial configuration, shapes, and identities of array items in repeated search (Chun \& Jiang, 1998, 1999; Shiffrin \& Schneider, 1977); target and distractor properties that produce a short-term influence on subsequent search trials (Maljkovic \& Nakayama, 1994, 1996); the positions of rejected distractors during a single search event (Klein, 1988; Klein \& MacInnes, 1999; Kristjánsson, 2000; Peterson, Kramer, Wang, Irwin, \& McCarley, 2001; Takeda \& Yagi, 2000); the positions of targets in multitarget search (Gibson, Li, Skow, Brown, \& Cook, 2000); and the visual form of rejected distractors (Castelhano \& Henderson, 2001; see Shore \& Klein, 2000, for a review of memory in visual search). Most relevant to the present argument are the results of Castelhano and Henderson (2001). In their study, participants searched for target objects in photographs of real-world scenes. After presenting all the scenes, memory for the visual form of earlier distractor objects was examined in a surprise test. Participants were significantly above chance on forced-choice orientation and token decisions, demonstrating preserved visual memory under incidental encoding conditions and for objects that were never the search target. Thus, evidence is accumulating to indicate robust visual memory for examined items in search.

\section{What Causes Change Blindness?}

Change blindness is defined as the failure to detect changes that are introduced into a scene or other visual image during some form of visual disruption, such as a saccadic eye movement or a brief interstimulus interval (ISI). Under these circumstances, a change cannot be detected directly (e.g., by perceiving motion); instead, change detection depends on comparing information stored in memory from the initial scene with perceptual information from the changed scene.

Because change detection requires the retention of visual information in memory, it is important to review briefly the literature on visual memory to establish a foundation for potential change blindness explanations. First, precise and complete sensory (i.e., iconic) representations are retained for only a very brief period after a stimulus event (e.g., Averbach \& Coriell, 1961; Di Lollo, 1980; Sperling, 1960). Such sensory representations cannot plausibly support memory across the temporal delays typically used in change detection studies. Second, sensory representations are not 
retained and integrated across eye movements to form a precise global image of a complex scene (Henderson, 1997; Irwin, 1991; Irwin, Yantis, \& Jonides, 1983; Rayner \& Pollatsek, 1983). Thus, sensory representation cannot plausibly support change detection across eye movements. Third, sensory information can be consolidated into more stable forms of visual memory (i.e., VSTM), but this transfer is limited, local, and highly dependent on attention, as demonstrated originally in partial-report paradigms (Averbach \& Coriell, 1961; Sperling, 1960) and then extended to transsaccadic memory (Irwin, 1992a; Irwin \& Gordon, 1998). Fourth, current evidence suggests that the capacity of VSTM across saccades and brief ISIs is limited to about four objects (Irwin, 1992a; Pashler, 1988; Vogel et al., 2001). Fifth, VSTM representations do not retain the full precision of sensory representation, such as absolute spatial coding (Irwin, 1991; Phillips, 1974) or a veridical representation of object contours (Henderson, 1997; Henderson \& Hollingworth, in press-c), but are detailed enough to code object token (Henderson \& Hollingworth, in press-a; Henderson \& Siefert, 2001), object orientation (Henderson \& Hollingworth, 1999b, in press-a; Henderson \& Siefert, 1999, 2001), and structural relationships between object parts (Carlson-Radvansky, 1999; Carlson-Radvansky \& Irwin, 1995). Such findings correspond nicely with evidence of viewpoint specificity and part decomposition in object recognition (Tarr \& Bülthoff, 1998), suggesting that visual object representations maintained in VSTM are likely to be similar to, if not the same as, representations that are stored in LTM and retrieved to support object recognition and categorization. Finally, visual representations can be retained in LTM for several minutes after stimulus presentation (Hollingworth \& Henderson, 2002) and for longer periods of $24 \mathrm{hr}$ (Standing et al., 1970) or even several weeks (DeSchepper \& Treisman, 1996).

In general, then, successful detection in a change blindness paradigm will depend on the initial encoding of a higher level visual representation from the target object, the retention of that representation either in VSTM or LTM, the retrieval of that representation, and the comparison of that representation to relevant perceptual information in the changed image. Change blindness could arise from limitations on any of these component processes: encoding failure, retention failure, or failures of retrieval and comparison.

Encoding failure. One of the clear causes of change blindness is failure to encode sufficient information from the initial scene image. First, encoding failure can occur if the changing region was not focally attended and fixated before a change was introduced. The transfer of visual information into memory is highly dependent on attention and fixation position. For example, Nelson and Loftus (1980) found that in an LTM paradigm, the detection of changes to objects in photographs was very near chance if there had not been a fixation within about $2^{\circ}$ of that object during study. To test the encoding failure hypothesis within an ongoing perceptual episode, Hollingworth and Henderson (2002) had participants view 3-D images of scenes and changed a target object, during a saccade, before the target been directly fixated. Under these conditions, change detection performance was no higher than the false alarm rate, whereas memory for previously fixated objects was often quite accurate.

In addition, even if an object is attended and some visual information is encoded from that object, participants may fail to encode sufficient information to detect a change because of limi- tations on viewing time. Hollingworth and Henderson (2002) found a positive correlation between fixation time on the target object before the change and subsequent change detection performance. Thus, attending an object is not sufficient to guarantee the encoding of all relevant information. Such encoding takes time, and if the target object is not attended long enough to encode sufficient information, the change will be missed. Similarly, in Experiment 2 of the present study, change detection performance was higher when a valid onset cue allowed participants to encode additional target information prior to the change.

Finally, participants may fail to encode sufficient information to support change detection because of limitations on the specificity of the higher level visual representations that are maintained in VSTM and across eye movements. A study by Henderson and Hollingworth (in press-c) illustrates this type of failure (see also Henderson, 1997). Participants viewed scene images, and each image was partially occluded by a set of vertical bars (as if the scene were viewed behind a picket fence). The bars shifted during saccades so that all visible regions of the scene became occluded and all occluded regions became visible. Thus, the entire scene was changed, ensuring that a change occurred wherever the participant was attending. Participants were told that the bars might shift during viewing; however, these changes were detected only $3 \%$ of the time-almost absolute change blindness. These data confirm that the object representations retained across saccades in natural scenes are abstracted away from the precise contours present in the image. ${ }^{5}$ A plausible explanation for this effect is that prior to a saccade, the higher level visual representation of the saccade target object completes shape information behind the occluding bars. On the next fixation after the change, a shape representation is again constructed that completes behind the (now shifted) bars. The pre- and postchange representations would therefore be highly similar, making change detection very difficult indeed, even though the two representations were constructed from complementary sets of information.

Retention failure. According to this possibility, sufficient information to detect a change is encoded from the initial scene, but that information is not retained to support change detection. Retention failure explanations have proposed roles for both decay and interference in the loss of previously encoded scene information. Decay as the mode of information loss is central to visual transience theories proposing that visual memory representations disintegrate immediately after attention is withdrawn from an object (Rensink, 2000; Wolfe, 1999); it is the withdrawal of attention that causes memory loss and not interference from subsequently encoded information. However, these theories also propose that there is considerable retention of nonattended information across views of a scene but that this information is unstable and highly prone to interference from subsequent perceptual input: New visual input overwrites visual information retained in memory from unattended regions of a scene (Rensink et al., 1997).

\footnotetext{
${ }^{5}$ Biederman and Cooper (1991) demonstrated that object recognition similarly relies on abstracted object representations. Line drawings of objects with half the contours deleted were presented at study, and later recognition performance was equivalent for identical images compared with images with the complementary set of contours.
} 
Retention failure explanations that depend on the assumptions of visual transience and overwriting have been by far the most popular accounts of change blindness phenomena to date (Rensink, 2000; Simons \& Levin, 1997; Wolfe, 1999). The primary data used to argue for retention failure have been the basic change blindness results obtained in the original flicker paradigm (Rensink et al., 1997). However, such change detection failure does not provide evidence to differentiate between the three potential causes of change blindness: Such effects could arise from retention failure, but they could also be caused by failures of encoding or failures of retrieval and comparison (see Simons, 2000).

The present evidence of robust visual memory after the withdrawal of attention, along with earlier evidence that visual memory remains accurate after a 5- to 30-min delay (Hollingworth \& Henderson, 2002), does not provide support for a retention failure explanation. However, these studies do leave room for such an explanation. In the present and related experiments, memory was tested for previously attended objects. Thus, these data eliminate the hypothesis that all visual information is lost when attention is withdrawn from an object, but they do not eliminate the possibility that some visual information is lost upon the withdrawal of attention, because detection performance, although generally good, was never perfect. In addition, the advantage for valid onset cues over invalid cues in Experiment 2 is consistent with the proposal that better visual information was retained for currently attended objects than for previously attended objects. However, this advantage could also be explained by increased encoding time in the valid onset condition and thus cannot provide strong evidence in favor of a retention failure hypothesis.

Failure of retrieval or comparison. Under this third possibility, sufficient information is encoded and retained from the initial image, but it is not retrieved and/or compared with perceptual information from the changed scene. The postcue conditions in the present study allowed participants to limit retrieval and comparison to the target and thus provided a measure of detection performance that minimized limitations on retrieval and comparison. The detection advantage for the postcue condition over the standard, no-postcue method demonstrates that limitations on retrieval and comparison are a major cause of change blindness effects. Simons et al.'s (2002) finding that participants can sometimes describe the visual characteristics of a deleted object (after failing to detect the deletion) suggests that retrieval and comparison failures play a significant role in incidental change detection paradigms as well.

The advantage in the postcue condition of Experiment 1 is similar to the partial-report advantage found in studies examining sensory persistence (e.g., Averbach \& Coriell, 1961; Sperling, 1960). This analogy should not be taken too far, however, because full report in sensory persistence studies is limited by rapid sensory decay. In contrast, higher level visual representations appear to be retained with little decay (Hollingworth \& Henderson, 2002). The postcue advantage in the present study likely derives from other sources. First, in the no-postcue condition, participants may not have attended to and fixated the target in the test scene prior to making a decision: Participants may not have been exhaustive in their comparison of test objects to memory. Second, participants may have been hesitant to base their report on partial information indicating a change, because other objects might provide better evidence in subsequent comparisons. This proposal is consistent with evidence that when changes are not explicitly reported, fix- ation durations on the changed object are nevertheless elevated (e.g., Hollingworth, Williams, \& Henderson, 2001). When the target is postcued, however, participants can apparently bring most, if not all, stored information to bear on the change decision. Finally, given that participants had to assess multiple objects to make a decision in the no-postcue condition, reduced detection performance relative to the postcue condition could be caused by the accumulation of decision error (see Vogel et al., 2001). Failures of retrieval and comparison could be viewed more generally as failures of decision processes. Error can enter into decision processes either because the relevant information was not adequately retrieved from memory, because the relevant perceptual information was not acquired from the test image, or because relevant memory and perceptual information were not adequately compared.

\section{Visual Memory Theory}

To account for accurate object memory in scenes, Hollingworth and Henderson (2002) proposed a visual memory theory of scene representation. This view rests on a foundation of earlier findings in the visual memory and eye movement literatures (as reviewed in the previous section; Henderson \& Hollingworth, 1999a; Irwin, 1992b). When attention and the eyes are oriented to a local object in a scene, in addition to low-level sensory processing, visual processing leads to the construction of higher level visual representations (which in turn lead to object recognition and the activation of conceptual and semantic information). The higher level visual representation of an attended object is maintained in VSTM and indexed to a position in a map coding the spatial layout of the scene, forming an object file (Henderson, 1994; Kahneman, Treisman, \& Gibbs, 1992). The object representation is then consolidated into LTM, retaining the position binding established in VSTM and forming a long-term memory object file (Hollingworth \& Henderson, 2002). When attention is withdrawn from an object, the VSTM representation decays as new information is entered into VSTM, leaving the LTM representation, which accumulates with other LTM representations from other previously attended objects. Just as the encoding of visual information into memory is local and dependent on attention, so is the retrieval of previously encoded visual representations. Evidence that retrieval depends on attention comes from the finding that change detection is often delayed until the target object is refixated later in viewing (Henderson \& Hollingworth, 1999b; Hollingworth \& Henderson, 2002).

This proposal places a good deal of the burden of scene representation on LTM. One might hesitate to associate LTM with the dynamic representation of visual information in scenes. But the following issues should be considered. First, the use of LTM in scene representation appears highly flexible and dynamic. In the present study, memory was tested during the active perception of a scene stimulus. In Hollingworth and Henderson (2002), accurate orientation and token discrimination was observed for previously attended objects even when a number of other objects had been fixated between the last fixation on the target and the test (making an explanation in terms of VSTM retention unlikely), but this delay was only a few seconds. If LTM accounts for such effects, then it is being used in a dynamic manner to support ongoing processing within a perceptual episode. Second, although visual 
representations in LTM are not visible in any meaningful sense, neither are representations in VSTM. Whereas brief sensory retention can be accompanied by visual phenomenology (i.e., visible persistence), visual retention across longer ISIs (when VSTM is required) is not accompanied by such experience. Thus, neither VSTM nor LTM storage is likely to play much of a direct role in visual phenomenology. Finally, as reviewed above, it is likely that VSTM supports the same form of visual representation as LTM. Thus, in scene perception, LTM appears to be used dynamically to store visual representations from previously attended objects (over time spans of a few seconds to at least a few minutes) as other object information is entered into VSTM. In addition, the LTM representations are surprisingly robust and resistant to decay, with little information lost from that available in VSTM (Hollingworth \& Henderson, 2002)

The use of LTM is likely to explain the apparent difference between memory capacity in the present study and memory capacity in studies using abstract arrays of stimuli and relatively brief presentation durations, in which visual memory for an array appears to be limited to three or four objects (Irwin, 1992a; Pashler, 1988; Vogel et al., 2001). However, there are other factors that may also contribute to the difference, including the use of meaningful natural objects in the present study, the relatively low interobject similarity, and the presence of meaningful spatial and semantic context. Evidence suggesting that contextual information influences visual object memory comes from the finding that VSTM retrieval is poorer when the spatial configuration of objects is changed or when the context is removed (Jiang, Olson, \& Chun, 2000). In addition, objects that are semantically inconsistent with scene context are retained in memory more accurately than are consistent objects, both in VSTM (Hollingworth \& Henderson, 1999, 2000) and in LTM (e.g., Friedman, 1979). Moreover, memory for object position in the inhibition of return phenomenon is dependent on the continued presence of the search context (Klein \& MacInnes, 1999; Takeda \& Yagi, 2000). Current experiments in my laboratory are investigating the properties of scene context that might support the accurate and robust retention of visual object representations.

Some final clarifications are necessary. Visual memory theory concerns the nature of visual memory representation but does not address issues of visual phenomenology. There is little in this view that could explain why people might experience the visual world as complete and detailed, even across eye movements. Note, however, that the phenomenology of seeing a "complete and detailed visual world" may have been a little oversold (see Nöe, Pessoa, \& Thompson, 2000). One can easily demonstrate that visual experience is not detailed and complete across the visual field by fixating a word in a page of text and attempting to read even nearby words.

In addition, visual memory theory specifies what visual memory is capable of representing from complex scenes, but there may be situations in which these capabilities are not used. ${ }^{6}$ This distinction between visual memory competence and actual visual memory performance is important for understanding two areas of research. One class of change detection paradigm-an incidental change paradigm - introduces a change while the participant is engaged in another activity and is not aware that a change may occur (e.g., Simons \& Levin, 1998). Change detection is assessed after the event by questioning the participant. Although this method may shed light on the important issue of what people happen to represent in memory under real-world conditions, it cannot provide strong evidence about visual memory capabilities. In particular, change blindness results derived from these methods cannot be used to argue that visual memory is incapable of supporting detailed scene representations, because change detection failure could arise from the failure to use existing memory capacity.

A similar point can be made regarding research on visual memory during common real-world tasks. Ballard, Hayhoe, and colleagues (Ballard et al., 1995; Ballard, Hayhoe, Pook, \& Rao, 1997; Hayhoe, 2000; Hayhoe et al., 1998; Land \& Hayhoe, 2001; Land et al., 1999) have argued that visual memory during common, real-world tasks is typically limited to the attended information necessary to support moment-to-moment actions. This functionalist approach to vision is supported by evidence that fixation position is tied closely to the immediate task at hand (Ballard et al., 1995; Hayhoe, 2000; Land et al., 1999). Such claims are not necessarily inconsistent with visual memory theory, because they concern the memory strategies that participants use during visuomotor tasks rather than the capabilities of visual memory. In addition, it is clearly the case that visual selection is controlled by task demands and viewer goals (Yarbus, 1967), and thus under visual memory theory, the information encoded into visual memory should likewise vary as a function of task. However, although evidence of a tight coupling between motor task and fixation position demonstrates that fixation plays an important role in motor actions, it does not demonstrate that visual memory is limited to the immediate task. Further, other evidence from these paradigms suggests that information outside of the immediate task is often represented in memory. First, changes to the color of blocks not currently being manipulated results in increased fixation times following the change (Hayhoe et al., 1998). Second, participants in a tea-making study by Land et al. (1999) exhibited a "surveying behavior," in which they fixated objects that would be used only later, and subsequent localizations of objects showed evidence of memory derived from these familiarization behaviors. ${ }^{7}$ Thus, research on vision during real-world tasks is generally compatible with the proposals outlined here. And visual memory theory makes a clear prediction in these paradigms: If an object has been attended and used but has ceased to be immediately relevant to the task, participants should nonetheless demonstrate preserved visual memory for the object.

\section{Conclusion}

The data from this study provide strong evidence that visual representations from local objects accumulate in memory as the eyes and attention are oriented within a scene, forming, over time, a scene representation of sufficient detail to make subtle visual judgments, such as detecting changes in object orientation and

\footnotetext{
${ }^{6}$ Visual transience theories also concern the capabilities of visual memory. The claim in this view is not that people do not happen to retain visual representations after the withdrawal of attention but rather that people cannot retain visual representations after the withdrawal of attention.

${ }^{7}$ The ability of visual memory to guide real-world tasks was demonstrated vividly in a recent televised cooking program, in which the chef, after familiarizing himself with the locations of his ingredients, prepared an entire dish blindfolded.
} 
object token. Thus, change blindness is not caused by the inability to retain visual information in memory. Instead, change blindness appears to derive primarily from constraints on encoding, retrieval, and comparison processes, because when these constraints were lifted, change blindness was largely cured.

\section{References}

Averbach, E., \& Coriell, A. S. (1961). Short-term memory in vision. The Bell System Technical Journal, 40, 309-328.

Ballard, D. H., Hayhoe, M. M., \& Pelz, J. B. (1995). Memory representations in natural tasks. Journal of Cognitive Neuroscience, 7, 66-80.

Ballard, D. H., Hayhoe, M. M., Pook, P. K., \& Rao, R. P. (1997). Deictic codes for the embodiment of cognition. Behavioral \& Brain Sciences, 20, 723-767.

Becker, M. W., Pashler, H., \& Anstis, S. M. (2000). The role of iconic memory in change detection tasks. Perception, 29, 273-286.

Biederman, I., \& Cooper, E. E. (1991). Priming contour-deleted images: Evidence for intermediate representations in visual object recognition. Cognitive Psychology, 23, 393-419.

Carlson-Radvansky, L. A. (1999). Memory for relational information across eye movements. Perception \& Psychophysics, 61, 919-934.

Carlson-Radvansky, L. A., \& Irwin, D. E. (1995). Memory for structural information across eye movements. Journal of Experimental Psychology: Learning, Memory, and Cognition, 21, 1441-1458.

Castelhano, M. S., \& Henderson, J. M. (2001, November). Eye movements, viewing task and scene memory. Paper presented at the 42nd Annual Meeting of the Psychonomic Society, Orlando, FL.

Chun, M. M., \& Jiang, Y. (1998). Contextual cueing: Implicit learning and memory of visual context guides spatial attention. Cognitive Psychology, $36,28-71$

Chun, M. M., \& Jiang, Y. (1999). Top-down attentional guidance based on implicit learning of visual covariation. Psychological Science, 10, 360365

Currie, C., McConkie, G., Carlson-Radvansky, L. A., \& Irwin, D. E. (2000). The role of the saccade target object in the perception of a visually stable world. Perception \& Psychophysics, 62, 673-683.

DeSchepper, B., \& Treisman, A. (1996). Visual memory for novel shapes: Implicit coding without attention. Journal of Experimental Psychology: Learning, Memory, and Cognition, 22, 27-47.

Deubel, H., \& Schneider, W. X. (1996). Saccade target selection and object recognition: Evidence for a common attentional mechanism. Vision Research, 36, 1827-1837.

Di Lollo, V. (1980). Temporal integration in visual memory. Journal of Experimental Psychology: General, 109, 75-97.

Fernandez-Duque, D., \& Thornton, I. M. (2000). Change detection without awareness: Do explicit reports underestimate the representation of change in the visual system? Visual Cognition, 7, 324-344.

Friedman, A. (1979). Framing pictures: The role of knowledge in automatized encoding and memory for gist. Journal of Experimental Psychology: General, 108, 316-355.

Gibson, B. S., Li, L., Skow, E., Brown, K., \& Cooke, L. (2000). Searching for one versus two identical targets: When visual search has memory. Psychological Science, 11, 324-327.

Grier, J. B. (1971). Nonparametric indexes for sensitivity and bias: Computing formulas. Psychological Bulletin, 75, 424-429.

Grimes, J. (1996). On the failure to detect changes in scenes across saccades. In K. Akins (Ed.), Perception: Vancouver studies in cognitive science (Vol. 5, pp. 89-110). Oxford, England: Oxford University Press.

Hayhoe, M. M. (2000). Vision using routines: A functional account of vision. Visual Cognition, 7, 43-64.

Hayhoe, M. M., Bensinger, D. G., \& Ballard, D. H. (1998). Task constraints in visual working memory. Vision Research, 38, 125-137.

Henderson, J. M. (1994). Two representational systems in dynamic visual identification. Journal of Experimental Psychology: General, 123, 410426.

Henderson, J. M. (1997). Transsaccadic memory and integration during real-world object perception. Psychological Science, 8, 51-55.

Henderson, J. M., \& Hollingworth, A. (1998). Eye movements during scene viewing: An overview. In G. Underwood (Ed.), Eye guidance in reading and scene perception (pp. 269-283). Oxford, England: Elsevier Science.

Henderson, J. M., \& Hollingworth, A. (1999a). High-level scene perception. Annual Review of Psychology, 50, 243-271.

Henderson, J. M., \& Hollingworth, A. (1999b). The role of fixation position in detecting scene changes across saccades. Psychological Science, 10, 438-443.

Henderson, J. M., \& Hollingworth, A. (in press-a). Eye movements and visual memory: Detecting changes to saccade targets in scenes. Perception \& Psychophysics.

Henderson, J. M., \& Hollingworth, A. (in press-b). Eye movements, visual memory, and scene representation. In M. A. Peterson and G. Rhodes (Eds.), Analytic and holistic processes in the perception of faces, objects, and scenes. New York: Oxford University Press.

Henderson, J. M., \& Hollingworth, A. (in press-c). Global transsaccadic change blindness during scene perception. Psychological Science.

Henderson, J. M., Pollatsek, A., \& Rayner, K. (1989). Covert visual attention and extrafoveal information use during object identification. Perception \& Psychophysics, 45, 196-208.

Henderson, J. M., \& Siefert, A. B. (1999). The influence of enantiomorphic transformation on transsaccadic object integration. Journal of Experimental Psychology: Human Perception and Performance, 25, 243-255.

Henderson, J. M., \& Siefert, A. B. C. (2001). Types and tokens in transsaccadic object identification: Effects of spatial position and leftright orientation. Psychonomic Bulletin \& Review, 8, 753-760.

Hoffman, J. E., \& Subramaniam, B. (1995). The role of visual attention in saccadic eye movements. Perception \& Psychophysics, 57, 787-795.

Hollingworth, A., \& Henderson, J. M. (1999). Object identification is isolated from scene semantic constraint: Evidence from object type and token discrimination. Acta Psychologica, 102, 319-343.

Hollingworth, A., \& Henderson, J. M. (2000). Semantic informativeness mediates the detection of changes in natural scenes. Visual Cognition, 7, 213-235.

Hollingworth, A., \& Henderson, J. M. (2002). Accurate visual memory for previously attended objects in natural scenes. Journal of Experimental Psychology: Human Perception and Performance, 28, 113-136.

Hollingworth, A., Schrock, G., \& Henderson, J. M. (2001). Change detection in the flicker paradigm: The role of fixation position within the scene. Memory \& Cognition, 29, 296-304.

Hollingworth, A., Williams, C. C., \& Henderson, J. M. (2001). To see and remember: Visually specific information is retained in memory from previously attended objects in natural scenes. Psychonomic Bulletin \& Review, 8, 761-768.

Horowitz, T. S., \& Wolfe, J. M. (1998, August 6). Visual search has no memory. Nature, 357, 575-577.

Horowitz, T. S., \& Wolfe, J. M. (2001). Search for multiple targets: Remember the targets, forget the search. Perception \& Psychophysics, 63, 272-285.

Irwin, D. E. (1991). Information integration across saccadic eye movements. Cognitive Psychology, 23, 420-456.

Irwin, D. E. (1992a). Memory for position and identity across eye movements. Journal of Experimental Psychology: Learning, Memory, and Cognition, 18, 307-317.

Irwin, D. E. (1992b). Visual memory within and across fixations. In K. Rayner (Ed.), Eye movements and visual cognition: Scene perception and reading (pp. 146-165). New York: Springer-Verlag.

Irwin, D. E., \& Andrews, R. (1996). Integration and accumulation of information across saccadic eye movements. In T. Inui and J. L. Mc- 
Clelland (Eds.), Attention and performance XVI: Information integration in perception and communication (pp. 125-155). Cambridge, MA: MIT Press.

Irwin, D. E., \& Gordon, R. D. (1998). Eye movements, attention, and transsaccadic memory. Visual Cognition, 5, 127-155.

Irwin, D. E., Yantis, S., \& Jonides, J. (1983). Evidence against visual integration across saccadic eye movements. Perception \& Psychophysics, 34, 35-46.

Irwin, D. E., \& Zelinsky, G. J. (2002). Eye movements and scene perception: Memory for things observed. Perception \& Psychophysics, 64, $882-895$.

Jiang, Y., Olson, I. R., \& Chun, M. M. (2000). Organization of visual short-term memory. Journal of Experimental Psychology: Learning, Memory, and Cognition, 26, 683-702.

Jonides, J. (1981). Voluntary versus automatic control over the mind's eye's movement. In J. B. Long \& A. D. Baddeley (Eds.), Attention and performance IX (pp. 187-203). Hillsdale, NJ: Erlbaum.

Kahneman, D., Treisman, A., \& Gibbs, B. J. (1992). The reviewing of object files: Object-specific integration of information. Cognitive Psychology, 24, 175-219.

Klein, R. (1988, August 4). Inhibitory tagging system facilitates visual search. Nature, 334, 430-431.

Klein, R. M., \& MacInnes, W. J. (1999). Inhibition of return is a foraging facilitator in visual search. Psychological Science, 10, 346-352.

Kowler, E., Anderson, E., Dosher, B., \& Blaser, E. (1995). The role of attention in the programming of saccades. Vision Research, 35, 18971916.

Kristjánsson, A. (2000). In search of remembrance: Evidence for memory in visual search. Psychological Science, 11, 328-332.

Land, M. F., \& Hayhoe, M. (2001). In what ways do eye movements contribute to everyday activities? Vision Research, 41, 3559-3565.

Land, M. F., Mennie, N., \& Rusted, J. (1999). Eye movements and the roles of vision in activities of daily living: Making a cup of tea. Perception, 28, 1311-1328.

Maljkovic, V., \& Nakayama, K. (1994). Priming of pop-out: I. Role of features. Memory \& Cognition, 22, 657-672.

Maljkovic, V., \& Nakayama, K. (1996). Priming of pop-out: II. The role of position. Perception \& Psychophysics, 58, 977-991.

McConkie, G. W., \& Currie, C. B. (1996). Visual stability across saccades while viewing complex pictures. Journal of Experimental Psychology: Human Perception and Performance, 22, 563-581.

Mitroff, S. R., Simons, D. J., \& Franconeri, S. L. (2002). The siren song of implicit change detection. Journal of Experimental Psychology: Human Perception and Performance, 28, 798-815.

Nelson, W. W., \& Loftus, G. R. (1980). The functional visual field during picture viewing. Journal of Experimental Psychology: Human Learning and Memory, 6, 391-399.

Nickerson, R. S. (1965). Short-term memory for complex meaningful visual configurations: A demonstration of capacity. Canadian Journal of Psychology, 19, 155-160.

Noë, A., Pessoa, L., \& Thompson, E. (2000) Beyond the grand illusion: What change blindness really teaches us about vision. Visual Cognition, 7, 93-106.

O'Regan, J. K. (1992). Solving the "real" mysteries of visual perception: The world as an outside memory. Canadian Journal of Psychology, 46, 461-488.

O'Regan, J. K., \& Noë, A. (in press). A sensorimotor account of vision and visual consciousness. Behavioral \& Brain Sciences.

O’Regan, J. K., Rensink, R. A., \& Clark, J. J. (1999, March 4). Change blindness as a result of "mudsplashes." Nature, 398, 34.

Pashler, H. (1988). Familiarity and the detection of change in visual displays. Perception \& Psychophysics, 44, 369-378.

Peterson, M. S., Kramer, A. F., Wang, R. F., Irwin, D. E., \& McCarley,
J. S. (2001). Visual search has memory. Psychological Science, 12, 287-292.

Phillips, W. A. (1974). On the distinction between sensory storage and short-term visual memory. Perception \& Psychophysics, 16, 283-290.

Posner, M. I., Snyder, C. R. R., \& Davidson, B. J. (1980). Attention and the detection of signals. Journal of Experimental Psychology: General, 109, $160-174$.

Rayner, K., McConkie, G. W., \& Ehrlich, S. (1978). Eye movements and integrating information across fixations. Journal of Experimental Psychology: Human Perception and Performance, 4, 529-544.

Rayner, K., \& Pollatsek, A. (1983). Is visual information integrated across saccades? Perception \& Psychophysics, 34, 39-48.

Remington, R. W., Johnston, J. C., \& Yantis, S. (1992). Involuntary attentional capture by abrupt onsets. Perception \& Psychophysics, 51, 279-290.

Rensink, R. A. (2000). The dynamic representation of scenes. Visual Cognition, 7, 17-42.

Rensink, R. A., O'Regan, J. K., \& Clark, J. J. (1997). To see or not to see: The need for attention to perceive changes in scenes. Psychological Science, 8, 368-373.

Schmidt, B. K., Vogel, E. K., Woodman, G. F., \& Luck, S. J. (2002). Voluntary and automatic attentional control of visual working memory. Perception \& Psychophysics, 64, 754-763.

Scholl, B. J. (2000). Attenuated change blindness for exogenously attended items in a flicker paradigm. Visual Cognition, 7, 377-396.

Shepard, R. N. (1967). Recognition memory for words, sentences, and pictures. Journal of Verbal Learning and Verbal Behavior, 6, 156-163

Shepherd, M., Findlay, J. M., \& Hockey, R. J. (1986). The relationship between eye movements and spatial attention. Quarterly Journal of Experimental Psychology: Human Experimental Psychology, 38(A), 475-491.

Shiffrin, R. M., \& Schneider, W. (1977). Controlled and automatic human information processing: II. Perceptual learning, automatic attending, and a general theory. Psychological Review, 84, 127-190.

Shore, D. I., \& Klein, R. M. (2000). On the manifestations of memory in visual search. Spatial Vision, 14, 59-75.

Simons, D. J. (1996). In sight, out of mind: When object representations fail. Psychological Science, 7, 301-305.

Simons, D. J. (2000). Current approaches to change blindness. Visual Cognition, 7, 1-16.

Simons, D. J., Chabris, C. F., Schnur, T. T., \& Levin, D. T. (2002). Evidence for preserved representations in change blindness. Consciousness \& Cognition, 11, 78-97.

Simons, D. J., \& Levin, D. T. (1997). Change blindness. Trends in Cognitive Sciences, 1, 261-267.

Simons, D. J., \& Levin, D. T. (1998). Failure to detect changes to people during a real-world interaction. Psychonomic Bulletin \& Review, 5, 644-649.

Sperling, G. (1960). The information available in brief visual presentations. Psychological Monographs, 74(11, Whole No. 498).

Standing, L. (1973). Learning 10,000 pictures. Quarterly Journal of Experimental Psychology, 25, 207-222.

Standing, L., Conezio, J., \& Haber, R. N. (1970). Perception and memory for pictures: Single-trial learning of 2500 visual stimuli. Psychonomic Science, 19, 73-74

Takeda, Y., \& Yagi, A. (2000). Inhibitory tagging in visual search can be found if search stimuli remain visible. Perception \& Psychophysics, 62, 927-934.

Tarr, M. J., \& Bülthoff, H. H. (1998). Image-based object recognition in man, monkey, and machine. Cognition, 67, 1-20.

Tarr, M. J., Bülthoff, H. H., Zabinski, M., \& Blanz, V. (1997). To what extent do unique parts influence recognition across changes in viewpoint? Psychological Science, 8, 282-289.

Tarr, M. J., Williams, P., Hayward, W. G., \& Gauthier, I. (1998). Three- 
dimensional object recognition is viewpoint dependent. Nature Neuroscience, 1, 275-277.

Theeuwes, J. (1991). Exogenous and endogenous control of attention: The effect of visual onsets and offsets. Perception \& Psychophysics, 49, 83-90.

Theeuwes, J., Kramer, A. F., Hahn, S., \& Irwin, D. (1998). Our eyes do not always go where we want them to go: Capture of the eyes by new objects. Psychological Science, 9, 379-385.

Vogel, E. K., Woodman, G. F., \& Luck, S. J. (2001). Storage of features, conjunctions, and objects in visual working memory. Journal of Experimental Psychology: Human Perception and Performance, 27, 92-114.

Williams, P., \& Simons, D. J. (2000). Detecting changes in novel 3D objects: Effects of change magnitude, spatiotemporal continuity, and stimulus familiarity. Visual Cognition, 7, 297-322.

Wolfe, J. M. (1999). Inattentional amnesia. In V. Coltheart (Ed.), Fleeting memories (pp. 71-94). Cambridge, MA: MIT Press.
Wolfe, J. M., Klempen, N., \& Dahlen, K. (2000). Postattentive vision. Journal of Experimental Psychology: Human Perception and Performance, 26, 693-716.

Yantis, S., \& Jonides, J. (1984). Abrupt visual onsets and selective attention: Evidence from visual search. Journal of Experimental Psychology: Human Perception and Performance, 10, 601-621.

Yantis, S., \& Jonides, J. (1990). Abrupt visual onsets and selective attention: Voluntary vs. automatic allocation. Journal of Experimental Psychology: Human Perception and Performance, 16, 121-134.

Yarbus, A. L. (1967). Eye movements and vision. New York: Plenum Press.

Received January 25, 2002

Revision received July 19, 2002

Accepted August 30, 2002

\section{American Psychological Association SUBSCRIPTION Claims INFORMATION}

Today's Date:

We provide this form to assist members, institutions, and nonmember individuals with any subscription problems. With the appropriate information we can begin a resolution. If you use the services of an agent, please do NOT duplicate claims through them and directly to us. PLEASE PRINT CLEARLY AND IN INK IF POSSIBLE.

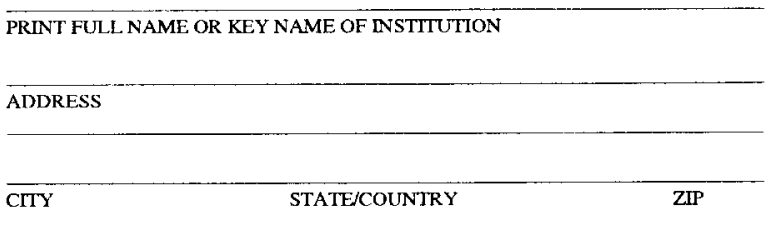

YOUR NAME AND PHONE NUMBER

TITLE
MEMBER OR CUSTOMER NUMBER (MAY BEFOUND ON ANY PAST ISSUE LABEL)

\begin{tabular}{l}
\hline DATE YOUR ORDER WAS MAIIED (OR PHONED) \\
PREPAID _ CHECK $\triangle$ CHARGE
\end{tabular}

(If possible, send a copy, front and back, of your cancelled check to help us in our research of your claim.)

VOLUME OR YEAR
ISSUES: MISSING DAMAGED
NUMBER OR MONTH

Thank you. Once a claim is received and resolved, delivery of replacement issues routinely takes 4-6 weeks.

(TO BE FILLED OUT BY APA STAFF)

DATE RECEIVED:

ACTION TAKEN:

STAFF NAME:
DATE OF ACTION:

INV. NO. \& DATE:

LABEL NO. \& DATE:

Send this form to APA Subscription Claims, 750 First Street, NE, Washington, DC 20002-4242

PLEASE DO NOT REMOVE. A PHOTOCOPY MAY BE USED. 